\title{
Dexamethasone-containing biodegradable superparamagnetic microparticles for intra-articular administration: Physicochemical and magnetic properties, in vitro and in vivo drug release
}

\author{
Nicoleta Butoescu ${ }^{\mathrm{a}, *}$, Olivier Jordan ${ }^{\mathrm{a}}$, Pierre Burdet ${ }^{\mathrm{b}}$, Pierre Stadelmann ${ }^{\mathrm{b}}$, Alke Petri-Fink $^{\mathrm{c}}$, \\ Heinrich Hofmann ${ }^{c}$, Eric Doelker ${ }^{a}$ \\ ${ }^{a}$ School of Pharmaceutical Sciences, University of Geneva, University of Lausanne, Geneva, Switzerland \\ ${ }^{\mathrm{b}}$ CIME, Swiss Federal Institute of Technology Lausanne, Lausanne, Switzerland \\ ${ }^{c}$ Powder Technology Laboratory, Institute of Materials, Swiss Federal Institute of Technology Lausanne, Lausanne, Switzerland
}

\section{A R T I C L E I N F O}

\section{Article history:}

Received 7 August 2008

Accepted in revised form 2 March 2009

Available online 20 March 2009

\section{Keywords:}

PLGA microparticles

Dexamethasone acetate

Superparamagnetic iron oxide nanoparticles (SPIONs)

Microscopy

SQUID

EELS

Drug release

Dorsal air pouch model

Arthritis

Intra-articular

\begin{abstract}
A B S T R A C T
Compared with traditional drug solutions or suspensions, polymeric microparticles represent a valuable means to achieve controlled and prolonged drug delivery into joints, but still suffer from the drawback of limited retention duration in the articular cavity. In this study, our aim was to prepare and characterize magnetic biodegradable microparticles containing dexamethasone acetate (DXM) for intra-articular administration. The superparamagnetic properties, which result from the encapsulation of superparamagnetic iron oxide nanoparticles (SPIONs), allow for microparticle retention with an external magnetic field, thus possibly reducing their clearance from the joint. Two molecular weights of poly(lactic-co-glycolic acid) (PLGA) were used, 12 and $19 \mathrm{kDa}$. The prepared batches were similar in size (around $10 \mu \mathrm{m}$ ), inner morphology, surface morphology, charge (neutral) and superparamagnetic behaviour. The SPION distribution in the microparticles assessed by TEM indicates a homogeneous distribution and the absence of aggregation, an important factor for preserving superparamagnetic properties. DXM release profiles were shown to be quite similar in vitro (ca. 6 days) and in vivo, using a mouse dorsal air pouch model (ca. 5 days).
\end{abstract}

(c) 2009 Elsevier B.V. All rights reserved.

\section{Introduction}

The use of carriers such as liposomes, nanoparticles or microparticles for intra-articular administration of drugs has gained wide acceptance over drug suspensions or solutions. The advantages of carriers lie in the fact that these delivery systems are, depending on their characteristics, cleared less rapidly from the joint, allowing for the active substance to be gradually released, and avoiding side effects such as crystal-induced arthritis [1]. The field of intra-articular-controlled drug delivery was pioneered by Ratcliffe et al. [2], who prepared albumin microspheres that could delay the clearance of a model drug from the joint, thus forming the rationale for intra-articular injections of microspheres. Moreover, the study also demonstrated that the clearance of this type of microparticle from the joint, known to be driven by synovial capillary and lymphatic transports, takes place within a few

\footnotetext{
* Corresponding author. School of Pharmaceutical Sciences, University of Geneva, 30 Quai Ernest Ansermet, Sciences II, 1211 Geneva 4, Switzerland. Tel.: +41 223796562 ; fax: +41 223796567.

E-mail address: Nicoleta.Butoescu@unige.ch (N. Butoescu).
}

days after injection. This study, with others [3,4], spurred the search for methods offering more controlled and prolonged delivery to the joint, focusing on ways to avoid rapid clearance.

Our approach can also be included among the studies in this field. Specifically, the aim of our work was to prepare and characterize dexamethasone acetate-containing magnetic poly(lactic-coglycolic acid) (PLGA) microparticles suitable for intra-articular injection for the treatment of arthritis or osteoarthritis. The rationale for formulating magnetic microparticles by encapsulating superparamagnetic iron oxide nanoparticles (SPIONs) into the polymer matrix is to enhance the retention of the microparticles in the joint with an external magnetic field. Compared to other papers reporting on the iron oxide encapsulation into polymer microparticles [5-8], rather restricted in number, our article describes the technology of co-encapsulation of iron oxide and a drug, process only very rarely seen in other works. Furthermore, in contrast to the encapsulation of iron oxide as oleic acid-coated SPIONs described in other reports, which, despite a high encapsulation content, could generate problems of toxicity, we encapsulate the SPIONs as an aqueous suspension, by a double emulsion-solvent evaporation method. The dose of dexamethasone that needs to 
be delivered to the joint was fixed at $1.2 \mathrm{mg} / \mathrm{kg}$, according to El Hakim et al. [9]. In a previous study [10], we optimized the formulation process of magnetic PLGA microparticles in terms of dexamethasone acetate (DXM) loading, encapsulating efficacy and magnetic retention by means of an experimental design. In particular we have demonstrated that the magnetic retention of this type of microparticle in a $0.8 \mathrm{~T}$ field was more than $80 \%$, making the microparticle suitable for clinical applications.

Considering that microparticle parameters such as size, shape, and polymer type influence the success of intra-articular local treatment of joint inflammation, we have carefully chosen microparticle specifications based on extensive literature data. Regarding particle size, small particles are easily phagocytosed, with reported limits ranging from the nanometer range up to a few micrometers. Howie et al. [11] reported that the macrophage response to particles larger than $5 \mu \mathrm{m}$ in diameter represents the approximate upper limit for phagocytosis. Horisawa et al. [12] used fluorescently labelled PLGA nanoparticles (mean diameter $265 \mathrm{~nm}$ ) and microparticles (mean diameter $26 \mu \mathrm{m}$ ) to evaluate synovial phagocytosis. As expected, nanoparticles were extensively phagocytosed and subsequently transported through the synovial membrane within 3-7 days. In contrast, $26 \mu \mathrm{m}$ mean diametermicroparticles were neither phagocytosed nor transported to the underlying synovial membrane. Furthermore, the $26 \mu \mathrm{m}$ diameter particles of Horisawa et al. triggered a granulation reaction with multinuclear giant cells. This tissular reaction could have resulted from a number of factors other than particle size, such as microparticle concentration in the articulation, polymer type or even particle shape. Concerning particle shape, Liggins et al. showed that irregular, milled chitosan particles induced joint inflammation despite the known articular biocompatibility of this biomaterial [13]. Because irregularly shaped microparticles are known to promote inflammation in diverse tissues, round-shaped microspheres are preferable for intra-articular delivery. Finally, with regard to the macromolecular carrier, Ratcliffe and co-workers [14] intra-articularly injected $1-10 \mu \mathrm{m}$ microparticles made of various polymers and showed that these materials elicited inflammation at different levels: severe for poly(butyl cyanoacylate), moderate for gelatine or poly(lactic acid) and weak for albumin. For our project, we decided to use PLGA as a polymer matrix for the microparticles, based on the demonstrated biocompatibility of PLGA with the articular tissues [12,13].

Considering the factors mentioned above, we prepared magnetic PLGA microparticles with a mean diameter of around $10 \mu \mathrm{m}$ for the local treatment of arthritis or osteoarthritis. The particles could possibly be maintained in the synovial space with an external magnet, ensuring a local release of the active substance, dexamethasone acetate. In this article, the optimized microparticles will be characterized by size, surface morphology, surface charge, SPION distribution inside the particle, magnetism, as well as in vitro and in vivo dexamethasone release.

\section{Materials and methods}

\subsection{Materials}

Poly(DL-lactide-co-glycolide) (PLGA) 75:25, inherent viscosity 0.16-0.24 dl/g, MW $19 \mathrm{kDa}$ (Resomer ${ }^{\circledR}$ RG 752S) and PLGA 50:50, inherent viscosity $0.16-0.24 \mathrm{dl} / \mathrm{g}, \quad \mathrm{MW} 12 \mathrm{kDa}\left(\operatorname{Resomer}^{\circledR} \mathrm{RG}\right.$ $502 S$ ), both of them having alkyl end groups, were provided by Boehringer Ingelheim (Ingelheim am Rhein, Germany). Dexamethasone 21-acetate (DXM) was purchased from Sigma-Aldrich (Epalinges, Switzerland). Poly(vinyl alcohol) (PVA, Mowiol ${ }^{\circledR}$ 4-88, hydrolysis degree $88 \%$ and mean molecular weight of $26 \mathrm{kDa}$ ) was a gift from Clariant GmbH (Frankfurt am Main, Germany). SPI-
ONs were prepared according to the method described by Chastellain and co-workers [15] and were used as an aqueous suspension, with an iron content of $10.3 \mathrm{mg} / \mathrm{mL}$, as assessed by atomic absorption.

\subsection{Preparation of microparticles}

The preparation of the microparticles was carried out by a double emulsion-solvent evaporation technique whereby SPIONs were encapsulated, and by simple emulsion solvent evaporation for the batches without SPIONs. The solvent mixture used was ethyl acetate with $10 \%$ ethanol, chosen based on preliminary DXM solubility studies using HPLC. The measured solubility in the mixture was $19 \mathrm{mg} / \mathrm{mL}$, but decreased to $15 \mathrm{mg} / \mathrm{mL}$ upon the addition of $300 \mathrm{mg} / \mathrm{mL}$ RG 502S. From these values, we selected a DXM concentration of $10 \mathrm{mg} / \mathrm{mL}$, in order to ensure proper solubilization and avoid crystal formation. Thus, the organic phase of the emulsion contained $300 \mathrm{mg} / \mathrm{mL}$ of polymer and $10 \mathrm{mg} / \mathrm{mL}$ DXM in ethyl acetate with $10 \%$ ethanol as co-solvent. Microparticles were prepared by emulsifying a volume of $3.5 \mathrm{~mL}$ organic phase containing $0.7 \mathrm{~mL}$ of ferrofluid $(10.3 \mathrm{mg} / \mathrm{mL}$ iron) with a sonicator for $10 \mathrm{~s}$. This primary emulsion was emulsified with $7 \mathrm{~mL}$ of $2 \%$ PVAL with a high speed homogenizer for $1 \mathrm{~min}$ at $8000 \mathrm{rpm}$. The final emulsion was then poured into $50 \mathrm{~mL}$ of $0.6 \%$ PVAL solution and stirred for $4 \mathrm{~h}$ at room temperature at $500 \mathrm{rpm}$. The resulting microparticles were separated by centrifugation, washed three times with deionised water and finally freeze-dried and stored at $4{ }^{\circ} \mathrm{C}$. Using the same method and the same technological parameters, a batch of "blank" microparticles was prepared for each of the two polymers (without DXM or SPIONs). Moreover, a batch of microparticles containing only DXM and another one of microparticles containing only SPIONs were also prepared using the two polymers by the simple emulsion technique and double emulsion technique, respectively (Table 1 ).

\subsection{Scanning electron microscopy (SEM)}

Scanning electron microscopy was performed on gold-coated freeze-dried samples (Balzers SCD 004 Sputter Coater) with a JEOL JSM-6400 microscope (JEOL Europe S.A., Croissy-sur-Seine, France) at an accelerating voltage of $15 \mathrm{kV}$ in order to determine the surface characteristics of the particles. For the visualization of the microparticle inner structure, cryo-fracturing SEM was used. Samples $\left(\sim 1 \mathrm{~mm}^{3}\right)$ were mounted on gold carrier plates and frozen by plunging into liquid propane (cooled by liquid nitrogen). Under liquid nitrogen, the carriers were then clamped to a specimen table, which was inserted into the cryo-unit (attached to a JEOL 6300 SEM) under a flow of dry nitrogen. To retain the samples in the fully frozen hydrated state they were kept at $-120^{\circ} \mathrm{C}$ and the sputtering process was started before fracturing. Then, the samples were fractured with a pre-cooled knife and coated with $10 \mathrm{~nm}$ of gold at a total pressure of $1.7 \times 10^{-2}$ mbar under pure argon gas. After completing the coating, high vacuum was re-established and the samples were transferred on-line to the SEM cryo stage. The SEM stage was cooled to $-150{ }^{\circ} \mathrm{C}$. SEM images were taken with an accelerating voltage of $15 \mathrm{kV}$.

\subsection{Particle size distribution}

The particle size was determined by laser diffraction with a Mastersizer apparatus (Malvern, Worcestershire, UK). The mean diameter used in this article represents the volume mean diameter, $D[4 ; 3], D[0.1], D[0.5]$ and $D[0.9]$ stand for the size of particle at which $10 \%, 50 \%$ and $90 \%$ of the sample are below this size in volume or in number. In addition, the span was calculated as follows: 
Table 1

Preparation of the eight batches of microparticles.

\begin{tabular}{lllll}
\hline Polymer & SPIONs & DXM & Technique & Batch number \\
\hline RG 502S & - & - & Simple emulsion & A1 \\
& + & - & Double emulsion & B1 \\
& - & + & Simple emulsion & C1 \\
& + & + & Double emulsion & D1 \\
RG 752S & - & - & Simple emulsion & A2 \\
& + & - & Double emulsion & B2 \\
& - & + & Simple emulsion & C2 \\
& + & + & Double emulsion & D2 \\
\hline
\end{tabular}

Span $=\frac{D[0.9]-D[0.1]}{[0.5]}$

2.5. Transmission electron microscopy (TEM) and energy-dispersive $X$ ray spectroscopy (EDX)

Thin sections ( $40 \mathrm{~nm}$ ) of samples embedded in epoxy resin were cut with an ultramicrotome and placed on a copper grid covered by a carbon film. The sections were observed with a transmission electron microscope Philips CM 30 at $300 \mathrm{kV}$. Chemical analyses were performed by energy-dispersive X-ray spectroscopy.

\subsection{Electron energy loss spectroscopy (EELS)}

In order to determine the oxidation state of the iron in the microparticles, electron energy loss spectroscopy was used. The experiment was performed on the same sections that underwent TEM and EDX with a Hitachi HF-2000 equipped with a field emission gun operated at $200 \mathrm{kV}$. The samples were cooled to liquid nitrogen temperature to prevent beam-induced contamination. The EEL spectra were recorded using a Gatan 666 parallel detection EEL spectrometer with an energy resolution of $0.5 \mathrm{eV}$ and a maximum dispersion of $0.05 \mathrm{eV}$ per channel.

\subsection{Zeta potential}

The zeta potential was measured with a Zetasizer 3000HS (Malvern, Worcestershire, UK) by electrophoretic mobility on suspensions of microparticles in $0.001 \mathrm{M} \mathrm{NaCl}$ with $0.1 \mathrm{M} \mathrm{HCl}$ or $0.1 \mathrm{M}$ $\mathrm{NaOH}$ as $\mathrm{pH}$ modifiers, and represent the average of five measurements.

\subsection{Differential scanning calorimetry (DSC)}

DSC study was performed with a DSC Seiko 220C (LabPlus, Bubikon, Switzerland) in sealed aluminium pans by heating samples at $10^{\circ} \mathrm{C} / \mathrm{min}$ in the $20-300^{\circ} \mathrm{C}$ range, cooled at $20^{\circ} \mathrm{C}$ and then reheated at $100{ }^{\circ} \mathrm{C}$. The instrument was calibrated using indium.

\subsection{X-ray diffraction (XRD)}

The XRD measurements, having the purpose of studying the crystallinity of DXM and of the SPIONs inside the microparticles, were conducted in an X-ray diffractometer (Philips PW1820) operating with $\mathrm{Cu} \mathrm{K} \alpha 1$ and $\mathrm{K} \alpha 2 \mathrm{X}$ radiations. The scans were recorded in the $2 \theta$ range of $10^{\circ}$ and $100^{\circ}$.

\subsection{Superconducting quantum interference device (SQUID)}

The magnetization was measured in a Quantum Design MPMS5 SQUID (Palaiseau, France) magnetometer, using a scan length of $4 \mathrm{~cm}$. We performed hysteresis loop measurements at $T=300 \mathrm{~K}$, up to 3000 Oe. We characterized the quality of the superparamagnetic behaviour from the hysteresis between the ascending and descending branches of the magnetization vs. the applied field plot and from the remanent magnetization value.

\subsection{Drug loading}

DXM loading of batches C1, D1, C2 and D2 was measured by HPLC after solubilization of the particles in acetonitrile. The suspension obtained after solubilization was centrifuged at $21,000 \mathrm{rpm}$ in order to separate the insoluble SPIONs (batches D1 and D2) from the DXM-containing solution. The supernatant was analyzed using a 100-5 C18 Nucleosil column (250/4.6, Macherey Nagel), an acetonitrile: water $1: 1$ mobile phase, a flow rate of $0.8 \mathrm{~mL} / \mathrm{min}$ and detection at $254 \mathrm{~nm}$ in order to assess DXM quantity.

\subsection{In vitro drug release}

In vitro DXM release was studied only on the batches containing both DXM and SPIONs (D1 and D2). In practical terms, 10-15 mg of microparticles were placed in $50 \mathrm{~mL}$ of $15 \mathrm{mM}$ PBS at pH 7.4 with $2 \mathrm{mM}$ sodium azide as a preservative and $0.5 \%$ polysorbate 80 as a solubility enhancer. The quantity of particles was adjusted in order to maintain sink conditions throughout the whole experiment. The suspension was circularly shaken at $120 \mathrm{rpm}$ and $37^{\circ} \mathrm{C}$ in a GFL3033 shaker (Burgwedel, Germany). At defined time intervals, aliquots of $0.5 \mathrm{~mL}$ were withdrawn and centrifuged to separate microparticles, which were returned to the release medium. Supernatants were assayed for DXM using HPLC. The withdrawn volume was replaced with fresh PBS. All experiments were performed in triplicate. At the end of the experiment, microparticles were separated, washed three times with purified water, lyophilised and viewed under a SEM to assess for signs of degradation.

\subsection{In vivo drug release}

The in vivo release study was performed using a dorsal air pouch model in mice [16-18]. C57Bl/6 mice (purchased from Harlan, France), aged between 8 and 10 weeks, were injected subcutaneously in the back with $4 \mathrm{~mL}$ of air, under isofluorane anaesthesia. The next day $0.5 \mathrm{~mL}$ of $2 \%$ sodium carmellose solution (carboxymethylcellulose sodium salt, low viscosity, SigmaAldrich, Epalinges, Switzerland) was injected into the pouch in order to accelerate exudative inflammation. Three days later, $0.5 \mathrm{~mL}$ of $10 \mathrm{mg} / \mathrm{mL}$ particle suspension in PBS was injected into the pouch. Five mice were sacrificed by $\mathrm{CO}_{2}$ inhalation at each time point (1, 2, 3 and 6 days after microparticle injection). The pouch was opened and the microparticles were withdrawn, washed three times with purified water, lyophilised and used for the HPLC assessment of the remaining DXM into the particles. Retrieved particles were also examined using a SEM to visualise possible degradation. All experimental procedures on animals were performed in compliance with the Swiss Federal Law on the Protection of the Animals, according to a protocol approved by the Swiss authorities.

\section{Results and discussion}

\subsection{Microparticle morphology}

The scanning electron micrographs (Fig. 1) reveal that the microparticles are spherical and exhibit a smooth surface without pores or channels. Furthermore, micrographs indicate that encapsulation of SPIONs and/or DXM leads to an increase in microparticle size. One possible explanation for this increase would be the decrease in viscosity resulting from the presence of the internal 

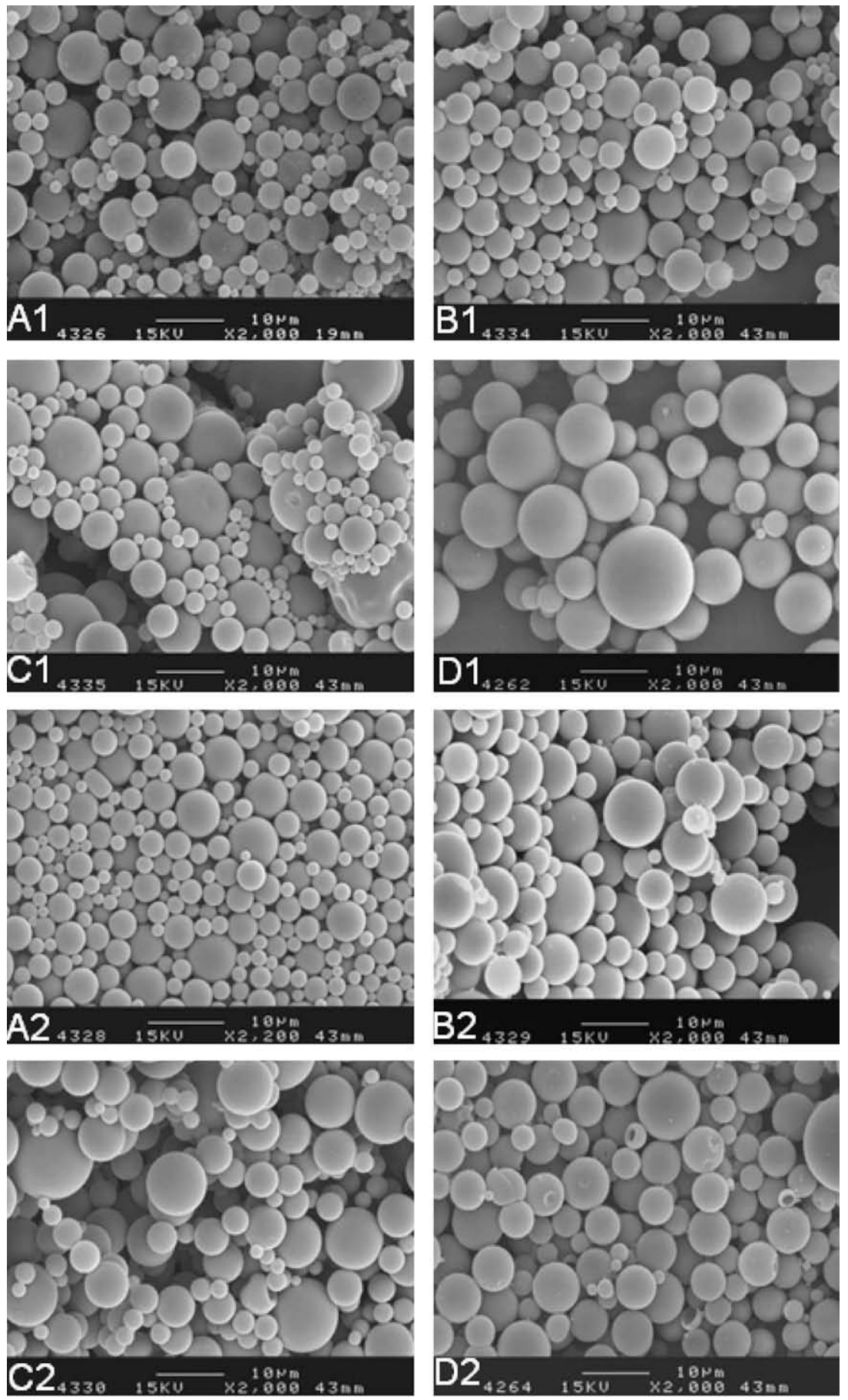

Fig. 1. Scanning electron photomicrographs of the RG 502S batches (A1, B1, C1, and D1) and the RG 752S batches (A2, B2, C2, and D2).

aqueous phase (SPION suspension). Similar observations were reported by Le Ray et al. for vancomycin-encapsulating poly( $\varepsilon$-caprolactone) microparticles [19].

In order to determine the inner structure of the microparticles and the details of their surface morphology, freeze-fracture scanning electron microscopy was performed. The images presented in Fig. 2 show that the microparticle inner structure can exhibit different morphologies. These different types of structures result from the fact that during the solvent evaporation and polymer precipitation the emulsion microdroplets coalesce, creating larger internal aqueous phase droplets [20]. The hardening of emulsion droplets with only one inner droplet of internal aqueous phase results in the formation of capsule-like structures. Moreover, during lyophilisation, the internal aqueous phase evaporates and the capsules can collapse, appearing as deflated balls (Fig. 1 batch D2). Fig. 2c shows another type of microparticle inner structure having a few small cavities. This structure results from the hardening of emulsion droplets having numerous aqueous phase droplets. Finally, in Fig. 2a, plain microparticles, resulting from the hardening of droplets containing no aqueous phase, are shown at a lower magnification than the two previous examples. These three types of internal structures are present in different proportions in all batches prepared by the double emulsion-solvent evaporation technique. Finally, images obtained with the 

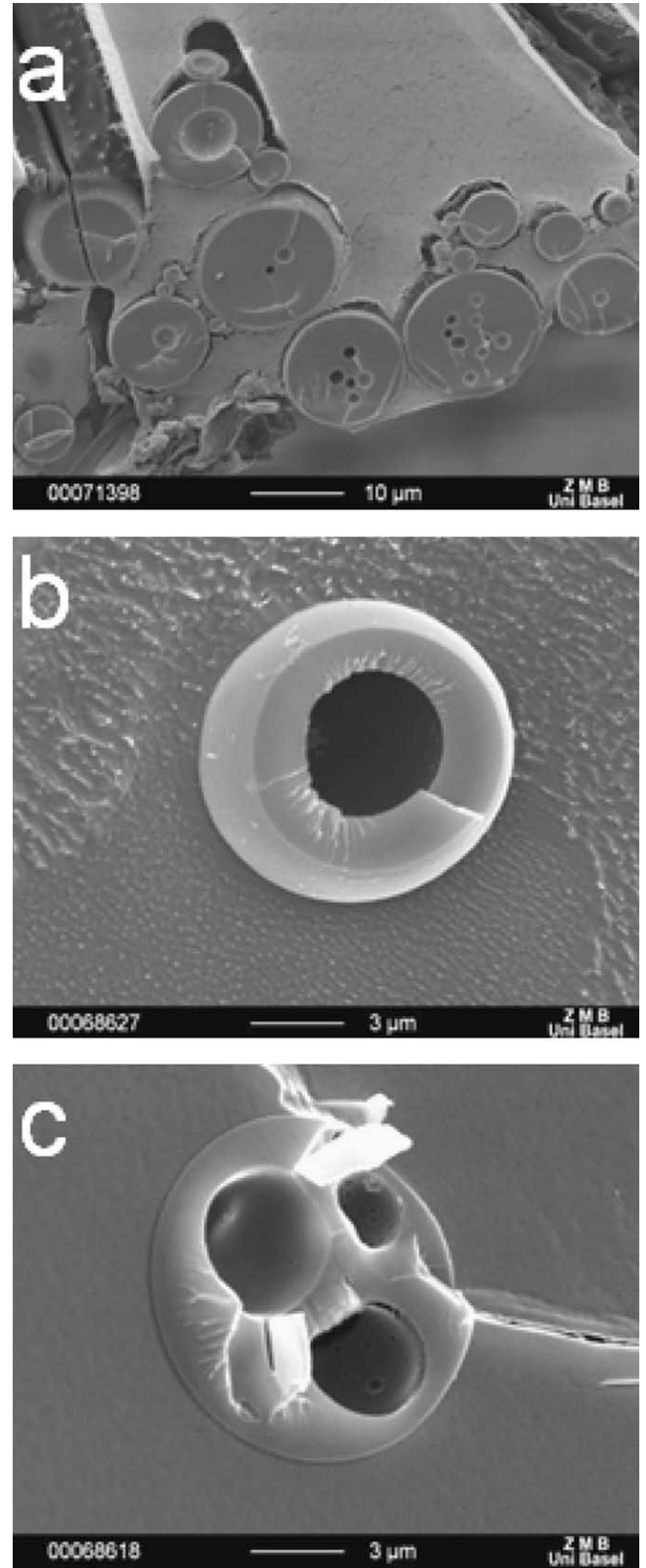

Fig. 2. Inner structure of the microparticles (batch D2). Different types of inner structure are present for the microparticles of the same batch. Similar images were recorded for all other batches prepared by the double emulsion-solvent evaporation technique.

cryo-fracture technique, at $5 \mathrm{kV}$ (compared to the micrographs in Fig. 1, acquired at $15 \mathrm{kV}$ ), allow us to state with certainty that the microparticle surface is smooth and without pores.

\subsection{Microparticle size distribution}

Larger particles were obtained with the RG 752S polymer, due to the higher viscosity of the $19 \mathrm{kDa}$ RG 752S polymer organic solution. Moreover, as shown by SEM, particle size also increased when DXM or SPIONs are encapsulated. The $D[4 ; 3]$ percentage of increase is $17 \%$ when SPIONs or DXM are encapsulated and 36\% when both substances are embedded into the RG 502S polymer microparticles. The percentage of increase was $10 \%, 66 \%$, and $12 \%$ when SPIONs, DXM and both chemical entities, respectively, are encapsulated into RG 752S microparticles. In general, the volume mean diameter, $D[4 ; 3]$, varies between $4.1 \mu \mathrm{m}$ for the blank RG 502S microparticles (batch A1) to $12.6 \mu \mathrm{m}$ for the SPION-encapsulating RG 752S microparticles (batch C2). In all cases, microparticle sizes are homogeneous with narrow and monomodal distributions (Table 2).

\subsection{SPION localization into the microparticles}

Fig. 3a shows the transmission electron micrograph of a capsule-like microparticle having peripheral localization of the SPIONs. At a higher magnification (Fig. 3b) it can be clearly observed that no significant nanoparticle aggregation is visible.

In order to confirm the chemical nature of the nanoparticles, EDX was performed on the circular zone as shown in Fig. 3. Additionally, a reference spectrum was acquired in a zone containing the EPON film without nanoparticles. The iron and oxygen peak areas increased by approximately five- and sixfold, respectively, compared to the reference. The data indicate that the nanoparticles are composed of iron and oxygen. However, the exact composition in these two elements cannot be precisely determined, both because EDX is not an accurate method to determine the light elements like oxygen or carbon and because the signal from the nanoparticles is situated at the quantification limit of the apparatus.

\subsection{Iron oxidation state in the microparticles}

The inner-shell excitation of the atoms with an electron beam leads to characteristic edges in the electron energy loss spectrum. The onset energies of each edge can be used to identify individual chemical elements to study bonding and local electronic properties of materials [21]. Fig. 4a represents the dark field scanning transmission electron micrograph of the SPIONs in the wall of a microparticle (visualized as white spots) which served for the measurement of the EEL spectrum, as shown in Fig. 4b. The EEL spectrum of the SPIONs involves the L3 and L2 edges of iron and the ratio L3/L2 of their relative intensities can be used to quantify the $\mathrm{Fe}^{3+} / \sum \mathrm{Fe}$ [22]. The measured ratio L3/L2 of $5.61 \pm 0.07$ is consistent with the previously published values for $\mathrm{Fe}^{3+}(5.64 \pm 0.10)$ and excludes the significant presence of magnetite $(5.00 \pm 0.09)$ [23]. Therefore, the iron oxide contained in the microparticles is in the form of maghemite.

\subsection{Microparticle surface charge}

The magnitude of the zeta potential, which determines the charge at the boundary of the diffuse region situated around the Stern layer, gives an indication on the stability of a colloidal system. The plot of the microparticle zeta potential as a function of $\mathrm{pH}$ (Fig. 5) shows that all batches, independently of the $\mathrm{pH}$, have zeta potential values between 5 and $-5 \mathrm{mV}$, leading to the conclusion that microparticle suspensions would not be very stable over time. For this reason, particles were stored as a lyophi- 
Table 2

The size distribution of all microparticle batches.

\begin{tabular}{|c|c|c|c|c|c|c|c|}
\hline \multirow[t]{2}{*}{ Batch number } & \multirow[t]{2}{*}{$D[4 ; 3](\mu \mathrm{m})$} & \multicolumn{3}{|l|}{ In volume } & \multicolumn{3}{|l|}{ In number } \\
\hline & & $D[0.1](\mu \mathrm{m})$ & $D[0.9](\mu \mathrm{m})$ & Span & $D[0.1](\mu \mathrm{m})$ & $D[0.9](\mu \mathrm{m})$ & Span \\
\hline A1 & 4.1 & 2.2 & 6.0 & 0.88 & 1.7 & 4.1 & 1.01 \\
\hline B1 & 4.8 & 2.2 & 7.9 & 1.19 & 1.5 & 4.2 & 1.20 \\
\hline $\mathrm{C} 1$ & 4.8 & 2.1 & 8.3 & 1.32 & 1.4 & 3.5 & 0.96 \\
\hline D1 & 5.6 & 2.6 & 9.0 & 1.19 & 1.6 & 5.0 & 1.40 \\
\hline A2 & 7.6 & 2.9 & 13.4 & 1.61 & 1.7 & 5.5 & 1.56 \\
\hline B2 & 8.4 & 3.8 & 14.3 & 1.38 & 1.7 & 6.0 & 1.69 \\
\hline $\mathrm{C} 2$ & 12.6 & 4.9 & 19.4 & 3.21 & 1.6 & 6.4 & 1.75 \\
\hline D2 & 8.5 & 3.3 & 14.8 & 1.52 & 1.8 & 5.8 & 1.51 \\
\hline
\end{tabular}
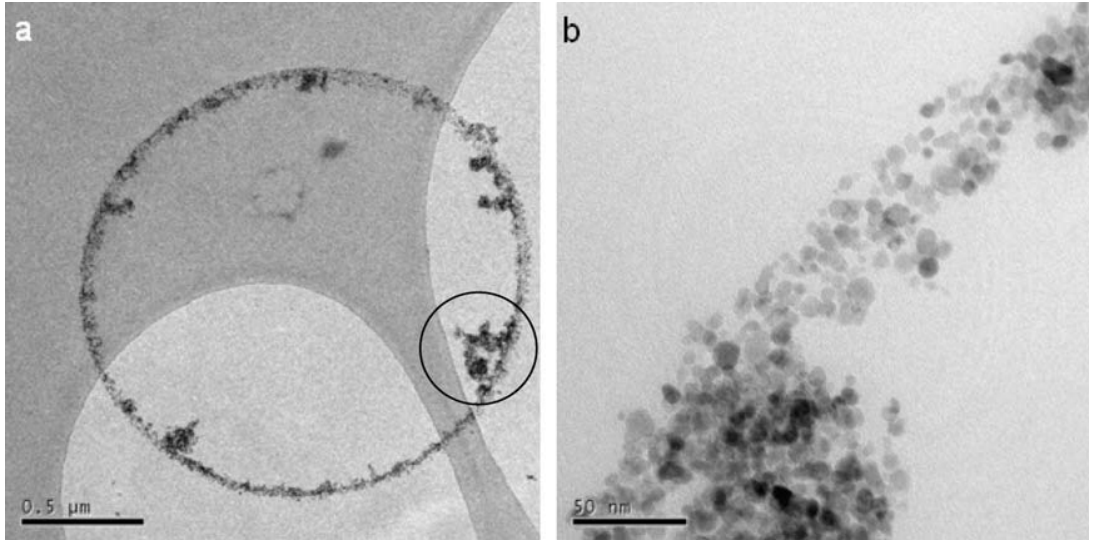

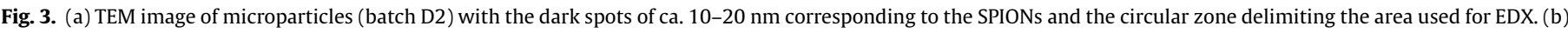
Higher magnification picture of a peripheral zone showing discrete SPIONs.
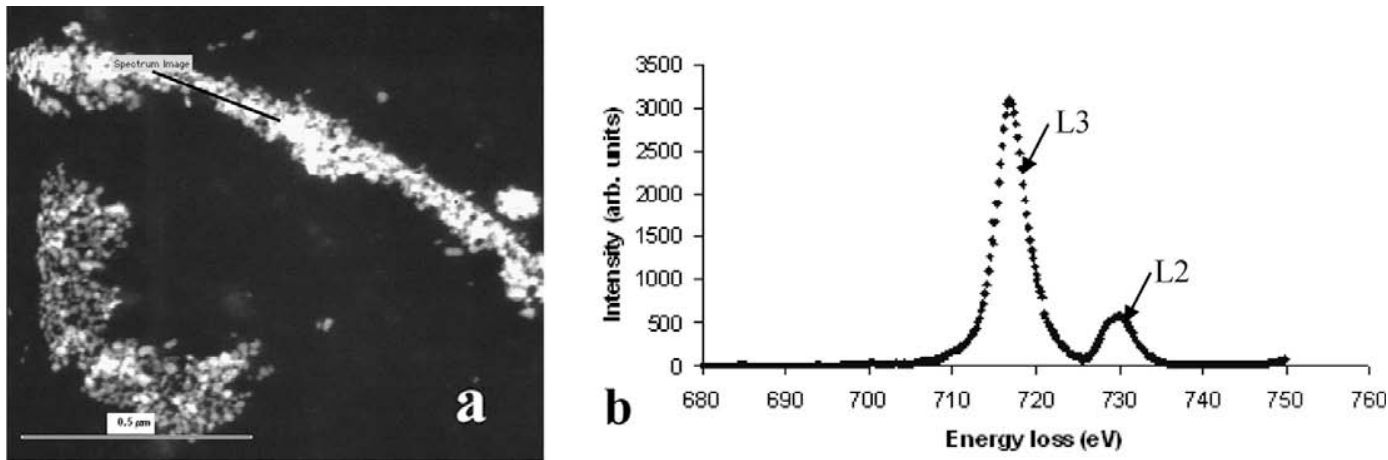

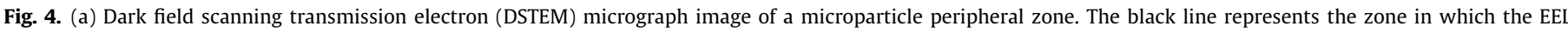

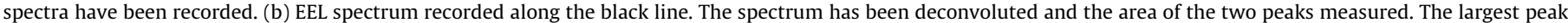
corresponds to the $\mathrm{Fe}^{3+}$ and the smaller one to $\mathrm{Fe}^{2+}$.

lized powder and resuspended upon need. From a physical point of view, Fig. 5 shows that the zeta potential of the SPIONs decreases from positive values to negative ones when the $\mathrm{pH}$ increases, with an isoelectric point at a $\mathrm{pH}$ around 7 , already reported in the literature [4]. The zeta potential of DXM crystals was also measured as a function of $\mathrm{pH}$ and, as can be seen in Fig. 5 , the values remain in the negative zone regardless of the $\mathrm{pH}$. These two observations, along with the fact that blank microparticles do not show any zeta potential variation with the $\mathrm{pH}$, allow us to conclude that neither DXM nor SPIONs are present at the surface of the microparticles. Finally, in addition to the stability problem, the surface charge of the particles represents an important factor in predicting the in vivo degree of interaction with the cell surface and thus the degree of microparticle uptake by the cells.

\subsection{DXM physical state in the microparticles}

DSC analysis of RG 752 S composite microparticles (Fig. 6a), a physical mixture of RG $752 \mathrm{~S}$ and DXM in the same proportions as in the microparticles (Fig. 6b), the polymer alone (Fig. 6c), and DXM alone (Fig. 6d) were carried out to determine the physical state of the DXM inside the particles as well as the changes in the thermal behaviour of the components once they were formulated as microparticles. Preliminarily, knowing that DXM exhibits polymorphism, we have determined by means of IR spectroscopy the form of the DXM used, which has been identified as Form II (data not shown), according to Kunhert-Brandstätter et al. [24]. The DSC thermogram of the microparticles (Fig. 6a) shows a peak at $50.5^{\circ} \mathrm{C}$ after a first heating cycle, corresponding to the enthalpy relaxation or the stress relief $[25,26]$. Moreover, no melting point 


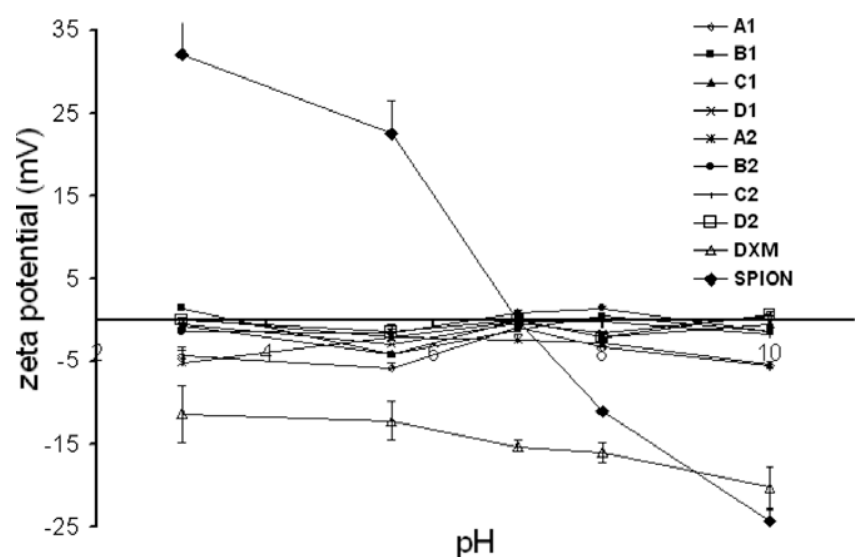

Fig. 5. Zeta potential of the microparticle batches, of DXM suspension and SPION suspension as a function of the $\mathrm{pH}$.

for DXM is present on the DSC curve, indicating that DXM is molecularly dispersed or in an amorphous state in the polymer matrix. On the thermogram of the physical mixture of polymer and DXM the peak corresponding to the enthalpy relaxation appears at $44.1^{\circ} \mathrm{C}$. On the same thermogram, the endothermic peak at $210.1^{\circ} \mathrm{C}$ results from the melting of DXM Form II. This is followed by the exothermic peak corresponding to the crystallization of Form I and the endothermic peak for the melting of the latter, at $222.4^{\circ} \mathrm{C}$, accompanied by some decomposition. On the thermogram of the polymer, the peak for the enthalpy relaxation appears at $45^{\circ} \mathrm{C}$. Finally, the thermogram of the DXM presents two endothermic peaks, one at $217.1^{\circ} \mathrm{C}$ and another at $234.3^{\circ} \mathrm{C}$, due to melting of the two polymorphic forms. From these four thermograms, it can be seen that the enthalpy relaxation peak is shifted to higher temperatures in the microparticle case compared to the polymer alone or to the physical mixture cases. This shift could be due to the presence of DXM or SPIONs into the microparticles that act as impurities and change the melting temperature. Moreover, the DXM melting temperature in the physical mixture is reduced compared to DXM alone, also resulting from the presence of impurities (e.g. in the polymer) that contaminate the DXM, disturbing its regular crystalline pattern. Furthermore, the presence of impurities is also the cause of the broadening and the depression of the DXM melting peak in the physical mixture of polymer and DXM. Upon a second heating of the samples up to $100^{\circ} \mathrm{C}$ at the same heating rates, the $T_{\mathrm{g}}$ of the polymer could be measured (Fig. 6e-g). In this case we could also see a shift, notably the $T_{\mathrm{g}}$ of the polymer inside the microparticles $\left(40.6^{\circ} \mathrm{C}\right)$ or in the physical mixture of polymer and DXM $\left(40.5^{\circ} \mathrm{C}\right)$ being about $3{ }^{\circ} \mathrm{C}$ higher than that of the polymer alone $\left(37.9^{\circ} \mathrm{C}\right)$. This shift also results from impurities. The same type of observations could me made for the RG 502S polymer (results not shown).

\subsection{Crystalline structure determination}

The crystallinity of the DXM alone, of the lyophilised SPIONs, and of the complete microparticles (batches D1 and D2) was studied by XRD. In Fig. 7, the XRD spectrum of pure DXM indicates a crystalline structure in the orthorhombic system. For the SPION sample, we obtained a pattern showing five characteristic peaks of the spinel structure of either maghemite or magnetite, in accordance with the previously published results [5]. In contrast, the spectra of both microparticle batches (not shown) do not display any crystalline peaks, most probably because the amounts of SPIONs and DXM are below the detection limits of the method.

\subsection{Magnetic properties}

Using SQUID, we measured the magnetic properties of the composite microparticles of batches D1 and D2, which have a SPION content of $0.9 \%$, and compared them with dried SPIONs as well as with a mixture of polymer and $0.9 \%$ SPIONs. The magnetization curves present negligible hysteresis loops, demonstrating that the iron oxide nanoparticles present in the samples have a superparamagnetic behaviour. The normalized curves to the SPION content in each sample superpose, leading to very similar saturation magnetizations. This indicates that the process of encapsulation does not change the magnetic properties of the SPIONs. This result is fundamental to future clinical applications, as it shows both that microparticles will not present remanent magnetization upon the suppression of the magnetic field and that the effective surface of SPIONs will not be diminished due to aggregation, in accordance

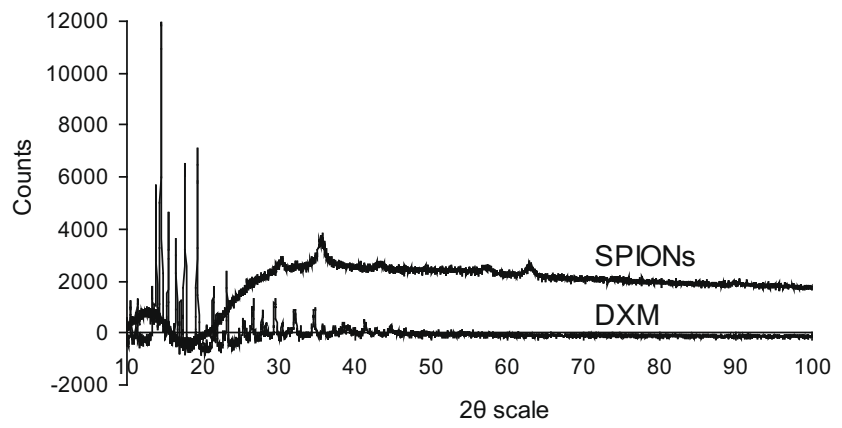

Fig. 7. The XRD spectra of DXM and SPIONs.
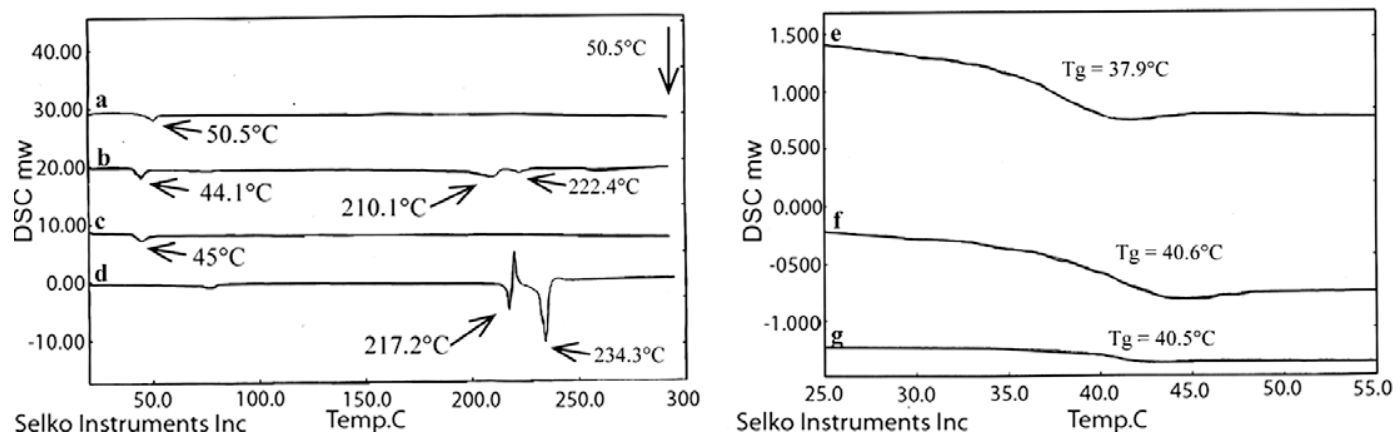

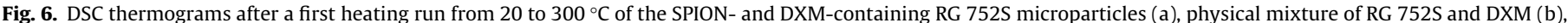

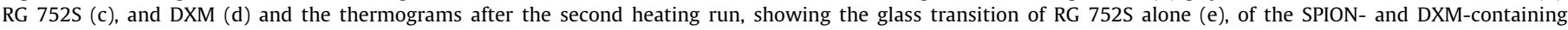
microparticles (f) and of the physical mixture of RG 752S and DXM ( $\mathrm{g}$ ). 
with the previous TEM observations. The value of the magnetization saturation of the lyophilized SPIONs of $51 \mathrm{emu} / \mathrm{g}$ represents the highest value among the studied samples. This result is comparable with the values obtained by other research teams. For example, Hamoudeh and co-workers [4] reported values of $70 \mathrm{emu} / \mathrm{g}$ for magnetite prepared by the co-precipitation method. Furthermore, the value of the saturation magnetization for a mixture of RG $752 \mathrm{~S}$ and lyophilized SPIONs was of $50.7 \mathrm{emu} / \mathrm{g}$ and of $45 \mathrm{emu} / \mathrm{g}$ for the batch D1 and of $46.7 \mathrm{emu} / \mathrm{g}$ for the batch D2 (Fig. 8). All these values were calculated for a magnetic field of $0.3 \mathrm{~T}$, which is typically the value of the magnetic field which should be clinically used.

\subsection{In vitro DXM release}

In vitro release studies of batches D1 and D2 (drug loading of $2.3 \%$ and $2.5 \%$, respectively, representing loading efficacy of more than $75 \%$ ) were conducted over 8 days. The release profiles (Fig. 9) demonstrate that the two batches present a sustained release during the studied period. It is important to note that microparticles of the batch D1 prepared with RG 502S completely released the active substance, while the microparticles prepared with RG 752 S released only $80 \%$ of the active substance during the 8 days of the experiment. This phenomenon could be due to the fact that, in the RG 752S microparticles (batch D2), DXM is more strongly associated to the polymer chains through hydrophobic interactions than in the RG 502S microparticles, due to a more important hydrophobicity of the first polymer compared to the second one. The curves were fitted to the semi-empirical model:

$M_{t}=M_{\infty}\left(1-\exp ^{-k t}\right)$

where $M_{t}$ and $M_{\infty}$ are the cumulative amount of drug released at time $t$ and infinite time, $k$ is a rate constant depending on the characteristics of the releasing system. For times longer than 3 days, the release rate slows to zero, indicating the end of the release process. The calculated half-lives corresponding to the two batches were quite similar, 0.8 days for batch D1 and 0.6 days for batch D2, with values for the rate constants of 0.9 days $^{-1}$ and 1.2 days $^{-1}$, respectively. In both cases, the correlation coefficient was higher than 0.989 .

Moreover, the molecular weight of the particles was determined by GPC after 21 days of in vitro release in order to test whether there is a diminution of the polymer molecular weight.

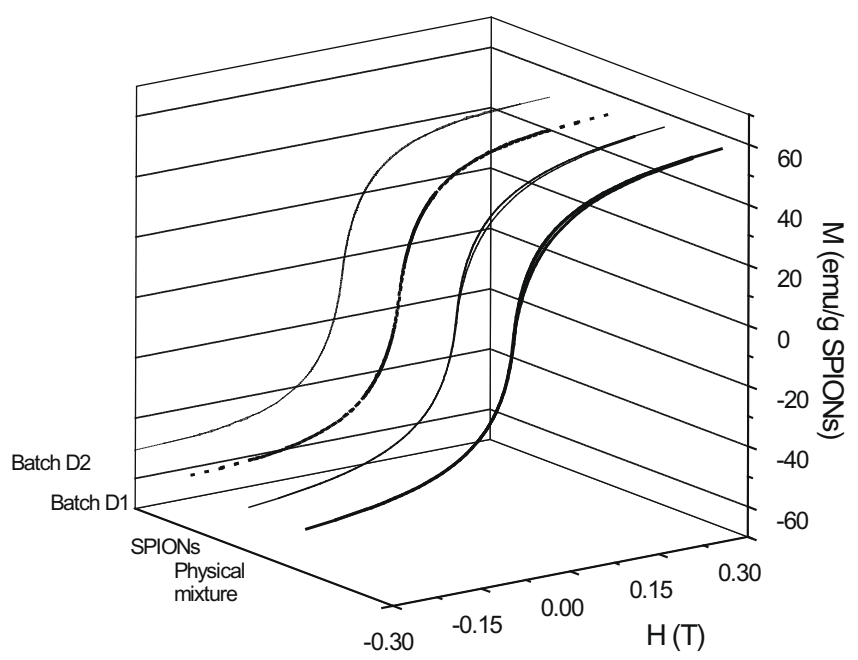

Fig. 8. Magnetograms of microparticle batches D1 and D2, of the SPIONs and of the physical mixture of SPIONs and polymer.

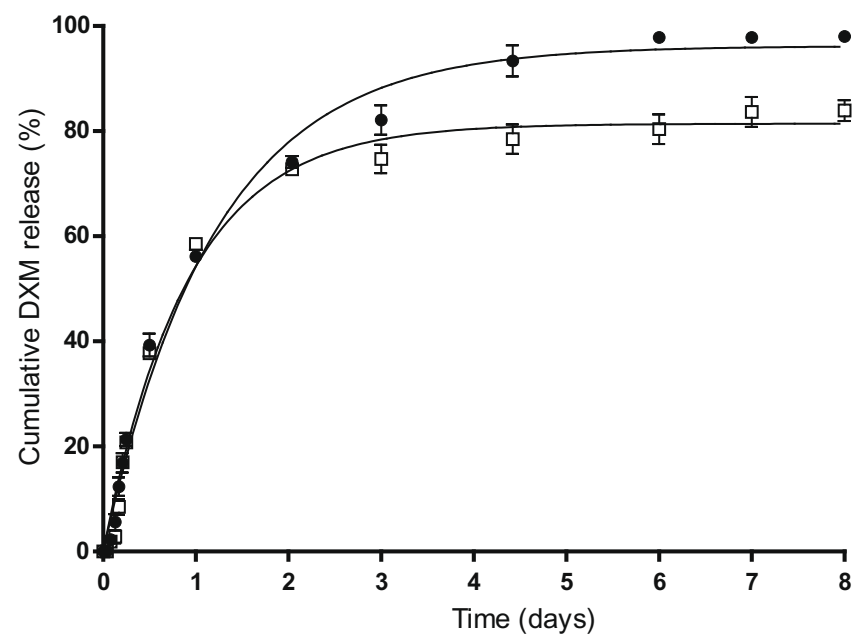

Fig. 9. In vitro drug release pattern of the batches D1 ( $\bullet$ ) and D2 ( $\square$ ). Error bars indicate standard error of the mean. Plain lines represent the first order kinetics fitting.

The obtained results demonstrate that the molecular weight remains constant for the duration of the experiment, proving that the DXM release process is mainly driven by diffusion. The fact that the polymer molecular weight does not change is consistent with SEM observations on the microparticles after 21 days of in vitro release (Fig. 11a and b). Microparticles in these images do not show signs of severe mechanical degradation, but only of slight surface erosion.

\subsection{In vivo DXM release}

The in vivo release of the encapsulated DXM was studied using a dorsal air pouch model in mice, generally used to mimic inflammatory conditions $[18,27]$. The purpose of this experiment was to assess how DXM is released from the microparticles in the presence of biological fluids and under inflammatory conditions. CollvilleNash and Lawrence [27] state that the maximal inflammatory response develops after a 7-day period. In our case, the inflammatory reaction was weak, due to the fact that we injected the microparticles only 4 days after the pouch formation.

The release profiles of the two investigated batches (Fig. 10) demonstrate that DXM is released from both batches in about 56 days. By fitting the curves to the same model as for the in vitro study, the release constants and the half-lives could be calculated. The correlation coefficient was higher than 0.995, showing that the mathematical model fitted the two curves well. For the batch D1, the release rate constant, $k$, had a value of 0.5 days $^{-1}$ and the half-life is 1.2 days, while for the batch D2 the release constant was 0.3 days $^{-1}$ and the half-life was 2.7 days. These values show that the DXM release from the RG 752S composite microparticles was slower than from RG 502S composite microparticles, possibly due to a tighter polymer network in the case of more hydrophobic RG $752 S$ polymer. Compared to the in vitro DXM release, the in vivo release was characterized by smaller release constants, and hence by larger half-lives, i.e. 1.5 and 4.3 times larger for batches D1 and $\mathrm{D} 2$, respectively. This difference could result from the high viscosity of environment in the pouch, due to both the presence of the carboxymethylcellulose solution and the very small quantity of exudate. Otherwise, the release profiles of the two batches have similar tendencies in vitro and in vivo. In both cases, the batch D1 released the active substance more rapidly than the batch D2 did. In order to mathematically assess whether the in vitro and in vivo release profiles for batches D1 and D2, respectively, were 


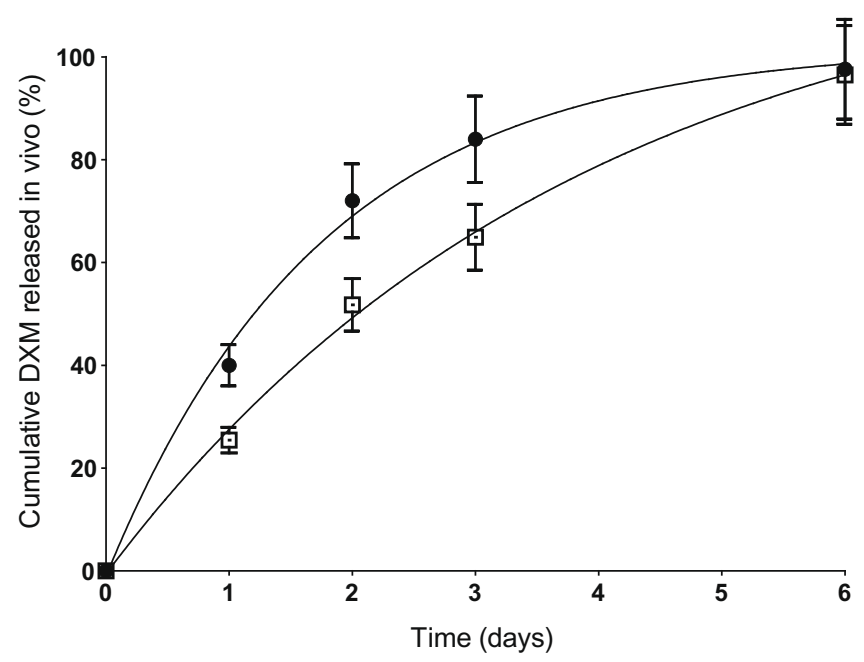

Fig. 10. In vivo drug release pattern of batches D1 (๑) and D2 ( $\square$ ). Plain lines represent the first order kinetics fitting. Error bars indicate standard error of the mean.

similar or not, the $f_{1}$ difference factor and $f_{2}$ similarity factor were calculated [28].

$f_{1}=\frac{\sum\left|R_{t}-T_{t}\right|}{\sum R_{t}} \times 100$

$f_{2}=50 \log \left[\frac{1}{\sqrt{1+\frac{1}{n} \sum\left(R_{t}-T_{t}\right)^{2}}} \times 100\right]$

where $n$ is the number of points in the dissolution profile, $R_{t}$ is the percent dissolved for the reference standard at each time period and $T_{t}$ is the percent dissolved for the test product at the same time per- iod, and log is logarithm base 10 . For equivalency, the $f_{1}$-value should be close to 0 (generally less than 15 ) and the $f_{2}$-value should be close to 100 (generally with values greater than 50). In our case, by comparing the in vitro and in vivo curves for batch D1 we obtained a value of 7 for $f_{1}$ and of 57 for $f_{2}$, while for batch D2 the values were 28 and 36 , respectively, for $f_{1}$ and $f_{2}$. These results prove that the in vitro and in vivo curves are similar in the case of batch D1 and that they are different in the case of batch D2, certainly due to the incomplete DXM release, in the latter case.

At the end of the experiment, microparticles were analyzed by SEM (Fig. 11c and d). The images show that the microparticles are only slightly eroded, proving that DXM is released by diffusion as in the case of the in vitro release. This result is also demonstrated by the fact that the molecular weight of the microparticles, assessed by GPC, does not change throughout the duration of the experiment. This can be expected, taking into consideration the fact that the acidic medium present in the exudate should degrade the polymer. However, the time of the experiment is probably too short for the hydrolysis of the polymer chains. Nevertheless, judging on the visual characteristics of the microparticle in Fig. 11, their rough surfaces may anticipate some signs of degradation that were not detected by gas chromatography.

In conclusion, we prepared magnetic RG 752S and RG 502S microparticles containing dexamethasone acetate for the intraarticular treatment of inflammatory diseases, such as arthritis. The encapsulation process did not affect the magnetic properties of SPIONs or their oxidation state. Moreover, DXM and SPIONs were completely embedded into the microparticles, as demonstrated by the zeta potential. The DXM microparticle loading complies with the necessary dose that can be administered into the articulation, which is of $1.2 \mathrm{mg} / \mathrm{kg}$ in rats or mice [9]. Complete microparticles made of both polymer types comply with the requirements for the intra-articular administering in terms of shape and size. Furthermore, in a previous article we have demonstrated that this type of microparticles have in vitro adequate
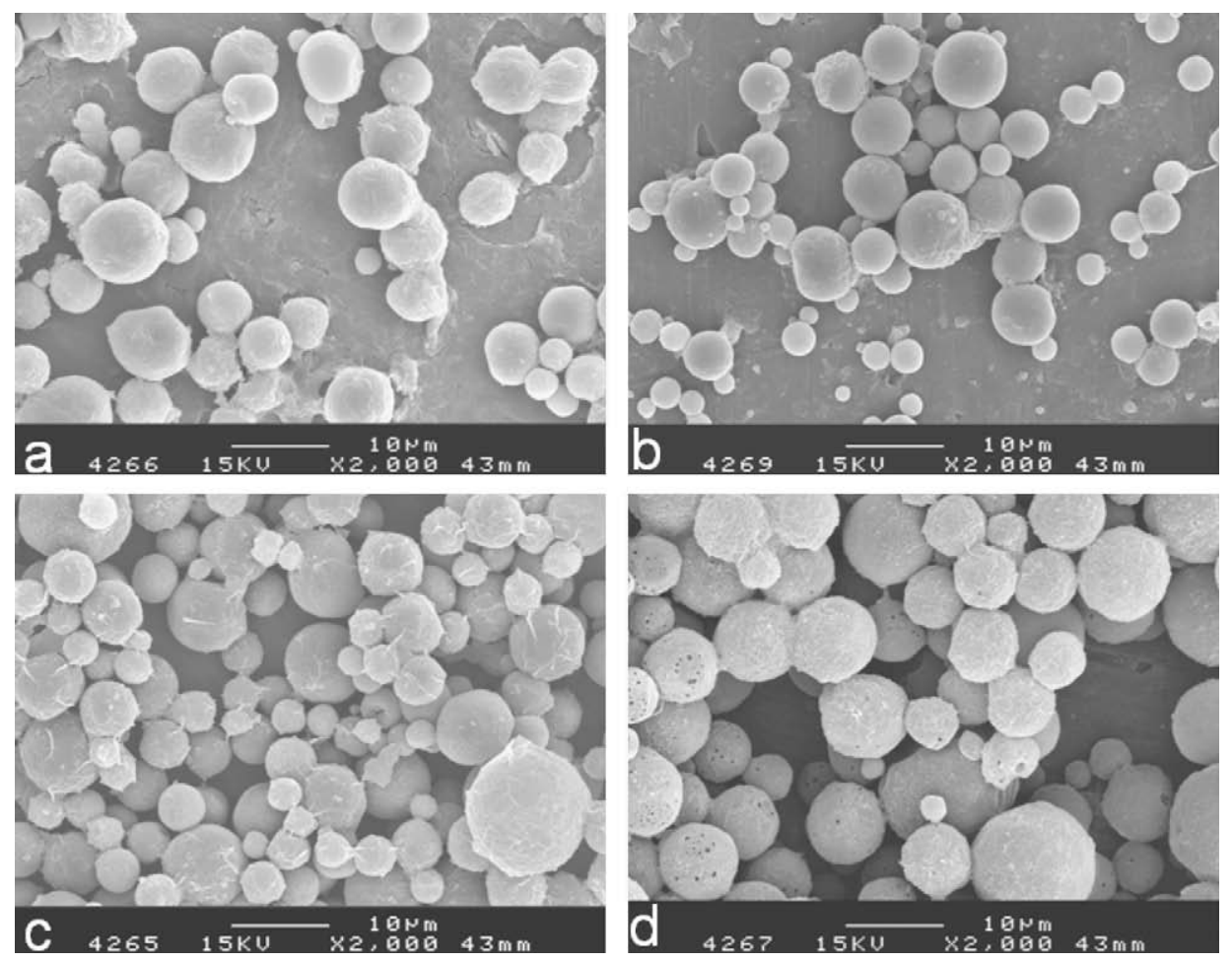

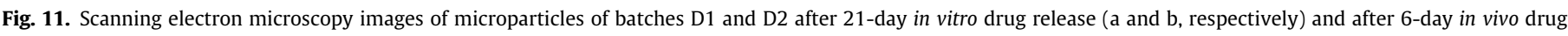
release (c and d, respectively). 
magnetic retention under the action of an external magnetic field. The in vitro and in vivo release patterns show similarity in terms of DXM release in the case of batches prepared with RG 502S polymer. An important aspect is that the in vivo release was performed in an inflammatory environment, such as in the case of arthritis, giving us an insight into the microparticle behaviour in the joint. As the physical characterization of the microparticles is done, studies of the interaction between the microparticles and the synovial cells are on going [29]. From the clinical point of view, the microparticles are being tested for their efficacy in a mouse model of antigen-induced arthritis and their retention in the joint will be studied in the absence or in the presence of an external magnet.

\section{Acknowledgements}

The authors would like to thank Mrs. Nathalie Boulens and Mrs. Catherine Siegfried (University of Geneva) for SEM images, Mr. Marcel Dueggelin (University of Basel) for freeze-fracture SEM, Dr. Cécile Hébert for helpful discussion on TEM and EELS, Mr. Jean-Luc Lorenzoni (University of Geneva) for XRD and Dr. Rolf Lortz for SQUID measurements.

\section{References}

[1] M.H. Ellman, M.A. Becker, Crystal-induced arthropathies: recent investigative advances, Curr. Opin. Rheumatol. 18 (2006) 249-255.

[2] J.H. Ratcliffe, I.M. Hunneyball, C.G. Wilson, A. Smith, S.S. Davis, Albumin microspheres for intra-articular drug delivery: investigation of their retention in normal and arthritic knee joints of rabbits, J. Pharm. Pharmacol. 39 (1986) 290-295.

[3] H. Thakkar, R.K. Sharma, A.K. Mishra, K. Chuttani, R.S.R. Murthy, Efficacy of chitosan microspheres for controlled intra-articular delivery of celecoxib in inflamed joints, J. Pharm. Pharmacol. 56 (2004) 1091-1099.

[4] S. Bozdag, S. Calis, H.S. Kas, M.T. Ercan, I. Peksoy, A.A. Hincal, In vitro evaluation and intra-articular administration of biodegradable microspheres containing naproxen sodium, J. Microencapsul. 18 (2000) 443-456.

[5] M. Hamoudeh, H. Fessi, Preparation, characterization and surface study of poly-epsilon caprolactone magnetic microparticles, J. Colloid Interf. Sci. 300 (2006) 584-590.

[6] M. Hamoudeh, A.A. Faraj, E. Canet-Soulas, F. Bessueille, D. Leonard, H. Fessi, Elaboration of PLLA-based superparamagnetic nanoparticles: characterization, magnetic behaviour study and in vitro relaxivity evaluation, Int. J. Pharm. 338 (2007) 248-257.

[7] L.N. Okassa, H. Marchais, L. Douziech-Eyrolles, K. Hervé, S. Cohen-Jonathan, E. Munnier, M. Sourcé, C. Linasssier, P. Dubois, I. Chourpa, Optimization of iron oxide nanoparticles encapsulation within poly(DL-lactide-co-glycolide) submicron particles, Eur. J. Pharm. Biopharm. 67 (2007) 31-38.

[8] R.A. Wassel, B. Grady, R.D. Kopke, K.J. Dormer, Dispersion of super paramagnetic iron oxide nanoparticles in poly(DL-lactide-co-glycolide) microparticles, Colloids Surf. A 292 (2007) 125-130.

[9] I.E. El Hakim, I.S. Abdel-Hamid, A. Bader, Tempromandibular joint (TMJ) response to intra-articular dexamethasone injection following mechanical arthropathy: a histological study in rats, Int. J. Oral Maxillofac. Surg. 34 (2005) 305-310.

[10] N. Butoescu, O. Jordan, A. Petri-Fink, H. Hofmann, E. Doelker, Co-encapsulation of dexamethasone 21-acetate and SPIONs into biodegradable polymeric microparticles designed for intra-articular delivery, J. Microencapsul. 25 (2008) 339-350.
[11] D.W. Howie, B. Manthey, S. Hay, B. Vernon-Roberts, The synovial response to intraarticular injection in rats of polyethylene wear particles, Clin. Orthop. Relat. Res. (1993) 352-357.

[12] E. Horisawa, K. Kubota, I. Tuboi, K. Sato, H. Yamamoto, H. Takeuchi, Y. Kawashima, Size-dependency of DL-lactide/glycolide copolymer particulates for intra-articular delivery system on phagocytosis in rat synovium, Pharm. Res. 19 (2002) 132-139.

[13] R.T. Liggins, T. Cruz, W. Min, L. Liang, W.L. Hunter, H.M. Burt, Intra-articular treatment of arthritis with microsphere formulations of paclitaxel: biocompatibility and efficacy determinations in rabbits, Inflamm. Res. 53 (2004) 363-372.

[14] J.H. Ratcliffe, I.M. Hunneyball, A. Smith, C.G. Wilson, S.S. Davis, Preparation and evaluation of biodegradable polymeric systems for the intra-articular delivery of drugs, J. Pharm. Pharmacol. 36 (1987) 431-436.

[15] M. Chastellain, A. Petri, H. Hofmann, Particle size investigations of a multistep synthesis of PVA coated superparamagnetic nanoparticles, J. Colloid Interf. Sci. 278 (2004) 353-360.

[16] D.L. Delano, M.C. Montesinos, P. D’Eustachio, T. Wiltshire, B.N. Cronstein, An interaction between genetic factors and gender determines the magnitude of the inflammatory response in the mouse air pouch model of acute inflammation, Inflammation 29 (2005) 1-7.

[17] E. Horisawa, T. Hirota, S. Kawazoe, J. Yamada, H. Yamamoto, H. Takeuchi, Y. Kawashima, Prolonged anti-inflammatory action of DL-lactide/glycolide copolymer nanospheres containing betamethasone sodium phosphate for intra-articular delivery system in antigen-induced arthritic rabbit, Pharm. Res 19 (2002) 403-410.

[18] S. Sud, S.-Y. Yang, C.H. Evans, P.D. Robbins, P.H. Wooley, Effects of cytokine gene therapy on particulate-induced inflammation in the murine air pouch, Inflammation 25 (2001) 361-372.

[19] A.-M. Le Ray, S. Chiffoleau, P. Iooss, G. Grimandi, A. Gouyette, G. Daculsi, C. Merle, Vancomycin encapsulation in biodegradable poly( $\varepsilon$-caprolactone) microparticles for bone implantation. Influence of the formulation process on size, drug loading, in vitro release and cytocompatibility, Biomaterials 24 (2003) 443-444

[20] I.D. Rosca, F. Watari, M. Uo, Microparticle formation and its mechanism in single and double emulsion solvent evaporation, J. Control. Release 99 (2004) 271-280.

[21] J. Mayer, Analysis of local structure, chemistry and bonding by electron energy loss spectroscopy, in: T.E. Weirich, J.L. Labar, X. Zou (Eds.), Electron Crystallography. Novel Approaches for Structure Determination of Nanosized Materials, Springer, Dordrecht, 2004, pp. 219-232.

[22] P.A. van Aken, V.J. Styrsa, B. Leibscher, A.B. Woodland, G.J. Redhammer Microanalysis of $\mathrm{Fe}^{3+} / \sum \mathrm{Fe}$ in oxide and silicate minerals by investigation of electron energy-loss near-edge structures (ELNES) at the Fe $M_{2,3}$ edge, Phys. Chem. Min. 26 (1999) 584-590.

[23] L. Cave, T. Al, D. Loomer, S. Cogswell, L. Weaver, A STEM/EELS method for mapping iron valence ratios in oxide minerals, Micron 37 (2006) 301-309.

[24] M. Kunhert-Brandstätter, P. Gasser, Solvates and polymorphic modifications of steroid hormones. III, Microchem. J. 16 (1971) 590-601.

[25] J. Rouse, F. Mohamed, C.F. van der Walle, Physical ageing and thermal analysis of PLGA microspheres encapsulating protein or DNA, Int. J. Pharm. 339 (2007) $112-120$.

[26] H.-H. Kausch, N. Heymans, C.J. Plummer, P. Decroly, Cohésion et état vitreux in: Matériaux polymères: propriétés mécaniques et physiques, Presses Polytechniques et Universitaires Romandes, Lausanne, 2001, pp. 47-88.

[27] P. Collville-Nash, T. Lawrence, Air-pouch models of inflammation and modifications for the study of granuloma-mediated cartilage degradation, in: P.G. Winyard, D.A. Willoughby (Eds.), Methods in Molecular Biology, Humana Press, Totowa, NJ, 2002, pp. 181-189.

[28] J.E. De Muth, Statistical tests for bioequivalence, in: J.E. De Muth (Ed.), Basics Statistics and Pharmaceutical Statistical Applications, Marcel Dekker, Basel, 1999, pp. 497-528.

[29] N. Butoescu, C.A. Seemayer, M. Foti, O. Jordan, E. Doelker, Dexamethasonecontaining PLGA superparamagnetic microparticles as carriers for the local treatment of arthritis, Biomaterials 30 (2009) 1772-1780. 